\title{
Visually Evoked Potentials in a Patient with a Fyodorov-Zuev Keratoprosthesis
}

\author{
Roy Schwartz Adiel Barak Hadas Newman \\ Department of Ophthalmology, Tel Aviv Medical Center, and Sackler Faculty of Medicine, \\ Tel Aviv University, Tel Aviv, Israel
}

\section{Key Words}

Keratoprosthesis · Visually evoked potentials

\begin{abstract}
Purpose: To describe a visually evoked potential (VEP) examination performed on a patient with a keratoprosthesis. Methods: We report the case of a 60-year-old patient with a Fyodorov-Zuev keratoprosthesis in the right eye complained of gradual visual deterioration in that eye. His past medical history consisted of failed corneal graft procedures due to corneal dystrophy and an Ahmed valve implantation due to secondary glaucoma. A clinical examination and an ultrasound demonstrated vitreal opacities. In order to assess the visual status, a flash VEP test was conducted. Results: VEP recorded from the right eye consisted of a broadened and poorly formed positive P1 wave, with a subnormal amplitude, but a normal latency. Consequently, the patient underwent a pars plana vitrectomy. Conclusion: This case demonstrates the viability of VEP exams in patients with keratoprostheses.

(c) 2015 S. Karger AG, Basel
\end{abstract}

\section{Background}

Keratoprosthesis is a surgical procedure where a severely damaged or diseased cornea is replaced with an artificial cornea. It is performed when corneal transplantation carries the risk of unsatisfactory results or when it is not feasible. Major indications include autoimmune-related corneal opacity and ulceration, chemical injury and corneal allograft failure.

Visually evoked potentials (VEPs) are electrical signals that are generated within the occipital lobe brain, in response to visual stimulation that begins at the retina.

The presence of a flash VEP response in case of media opacities is an important indicator of the visual status. Electrodiagnostic tests, such as electroretinogram (ERG), which eval-

KARGER 125is $\quad \begin{aligned} & \text { Roy Schwartz } \\ & 6 \text { Weizmann Street } \\ & \text { Tel Aviv } 64239 \text { (Israel) } \\ & \text { E-Mail royschwartz@gmail.com }\end{aligned}$


Schwartz et al.: Visually Evoked Potentials in a Patient with a Fyodorov-Zuev Keratoprosthesis

uate retinal function and Flash VEP, are important prognostic factors in cases of vitreous hemorrhage, cataract and corneal opacities [1-5].

To our knowledge, this is the first case reported in the literature describing VEP responses in a patient with a Fyodorov-Zuev keratoprosthesis.

\section{Case Report}

A 60-year-old healthy patient presented to our clinic with gradual visual deterioration over the past months in his right eye (RE). His ocular history included bilateral corneal dystrophy, symptomatic since age 23. In the RE, he had undergone several ocular procedures, including a few penetrating keratoplasty operations, cataract extraction and implantation of an Ahmed valve due to secondary glaucoma. His last procedure in the RE 5 years prior consisted of a Fyodorov-Zuev keratoprosthesis implantation, and postoperative visual acuity was documented as $20 / 100$. In the left eye (LE), he underwent a single corneal transplantation with a later graft failure, and a repeated corneal transplantation had been planned.

Upon presentation, his best-corrected visual acuity was $\mathrm{CF}$ at $1 \mathrm{~m}$ in the RE and $\mathrm{CF}$ at 2 $\mathrm{m}$ in the LE. His intraocular pressures were $16 \mathrm{~mm} \mathrm{Hg}$ and $15 \mathrm{~mm} \mathrm{Hg}$, respectively. An examination of the RE (fig. 1) revealed a corneal pannus and a central keratoprosthesis with an Ahmed implant behind it. The vitreous was hazy and did not allow a view of the posterior segment. In the LE, an opaque full-thickness corneal graft was seen with corneal neovascularization, blocking view of the posterior segment.

An ultrasound of the RE revealed mobile opacities and an attached retina. No other abnormalities were observed. An ultrasound of the LE revealed a clear vitreous and an attached retina.

The visual decrease in the RE was attributed to the vitreal opacities, and a pars plana vitrectomy was considered. In order to assess the visual status and operative prognosis, a flash VEP examination was performed. Although flash VEPs are less consistent between subjects (relative to spatially structured stimuli-evoked potentials), this test is mainly indicated in special circumstances, such as poor compliance and fixation or media opacities [6], and thus it was chosen in this case.

VEP recorded from the LE consisted of well-formed negative N1 and positive P1 waves, with normal implicit times and amplitudes. VEP recorded from the RE consisted of broadened and poorly formed N1 and P1 waves, with smaller amplitudes, but normal implicit times. VEP recorded from binocular stimulation resembled the LE VEP wave forms, but consisted of higher amplitudes in keeping with some RE contribution (fig. 2). An ERG was not performed due to the presence of the keratoprosthesis, which does not permit the placement of a corneal electrode.

Following the documentation of RE VEP responses with no implicit time delay, an endoscopic pars plana vitrectomy was scheduled. Core vitrectomy followed by peripheral vitrectomy were performed. During surgery, the retina appeared normal while the optic disc appeared pale with a high cup-to-disc ratio (fig. 3). An adherent membrane posterior to the keratoprosthesis was evident as well, which was cleared using vitrectomy.

On postoperative day 1 , visual acuity was $1 / 230$ OD, but it deteriorated 3 days later to light perception. On repeat ultrasound of the RE, a mild vitreous hemorrhage was seen as well as a small area of retinoschisis in the inferotemporal quadrant. At that point, the patient returned abroad to his home country for continued follow up. 
Schwartz et al.: Visually Evoked Potentials in a Patient with a Fyodorov-Zuev Keratoprosthesis

\section{Discussion}

To our knowledge, this is the first case report to describe VEP responses recorded from a patient with a keratoprosthesis.

The poor visual acuity upon presentation is attributed to several factors, including the presence of vitreal opacities, a retroprosthetic membrane and advanced glaucoma.

Patients with keratoprosthesis may develop vitreal opacities or a sterile vitritis during the lifetime of their implant. Vitreal opacities were documented in cases of the more common Boston keratoprosthesis (K-pro) [7, 8], but are also described in eyes with a FyodorovZuev keratoprosthesis, with cases of endophthalmitis and growth of a granulation tissue [9]. The development of a retroprosthetic membrane, which grows on the posterior surface of the optical cylinder of the implant, is another documented side effect of keratoprosthesis procedures, leading to a decreased visual acuity [9].

The prevalence of glaucoma in patients undergoing K-pro implantation ranges from 36 to $76 \%$ [10]. No data is available for the Fyodorov-Zuev keratoprosthesis. Between 14 and $28 \%$ of patients develop an elevated intraocular pressure (IOP) following K-pro surgery. De novo glaucoma has been reported to occur in $2-28 \%$ of patients after K-pro implantation, and up to $13 \%$ have required subsequent surgical interventions for the treatment of glaucoma. The diagnosis of glaucoma in K-pro patients is complicated because of a lack of a reproducible method for measuring their IOP. Although Tonopen measurements at the limbus may provide a rough assessment of the IOP, finger tension is the most commonly employed method of estimating a patient's IOP [10]. Our patient had a history of glaucoma and therefore necessitated a shunt procedure. During the vitrectomy, a pale disc with an increased cup-to-disc ratio was seen. Therefore, advanced glaucoma was probably a major contributor to his decreased vision, which was not fully appreciated before surgery, possibly due to inaccurate IOP measurements.

Despite successful vitrectomy, the patient's visual acuity did not improve and even deteriorated following the surgery. Possible explanations are the deterioration of the glaucoma following surgery and the mild vitreous hemorrhage documented per ultrasound. No retinal detachment or retroprosthetic membranes were evident. As the patient was not available for further follow-up, the current state of his visual acuity is unknown.

Electrodiagnostic tests such as ERG and Flash VEP are important prognostic factors in cases of media opacities, such as vitreous hemorrhage, cataract and corneal opacities [1]. Scherfig et al. [2] conducted VEPs in 116 diabetic eyes prior to vitrectomy. Delayed VEP responses with a positive peak appearing beyond $100 \mathrm{~ms}$ were associated with a smaller chance for vision improvement. Other studies have also claimed that VEP can be a good predictor of visual outcome in patients with vitreous hemorrhage $[3,4]$. In our patient, VEP waves from the RE were formed less sharply, but a positive P1 was evident with a normal implicit time (89 ms). Some RE contribution to the binocular VEPs was evident as well. As the flash VEP responses demonstrated the transmission of visual information from the RE to the visual cortex, they supported the decision to operate on the patient.

The combination of an ERG to evaluate retinal function and a VEP exam might be preferable to evaluate a visual status. Wendel et al. [5] compared preoperative flash VEPs and ERGs to postoperative findings in a series of 32 patients undergoing elective penetrating keratoplasty. They were able to predict the visual outcome in $92 \%$ of cases. In this case, ERG was not performed due to the corneal prosthesis, excluding corneal electrode placement.

In conclusion, this case demonstrates the feasibility of recording VEP responses in patients with keratoprosthesis. Complications such as vitreal opacities and retroprosthetic 
Schwartz et al.: Visually Evoked Potentials in a Patient with a Fyodorov-Zuev Keratoprosthesis

membranes are described. Visual improvement might be limited due to glaucomatous damage, which could be difficult to assess and control in patients with keratoprosthesis.

\section{Disclosure Statement}

None of the authors have any conflicts of interests regarding the material presented in this paper.

\section{References}

1 Galloway NR: Electrophysiological testing of eyes with opaque media. Eye (Lond) 1988;2:615-624.

-2 Scherfig E, Edmund J, Tinning S, Trojaborg W: Flash visual evoked potential as a prognostic factor for vitreous operations in diabetic eyes. Ophthalmology 1984;91:1475-1479.

-3 Tolentino FI, Schepens CL, Mackenzie Freeman H: Vitreoretinal Disorders: Diagnosis and Management. Philadelphia, W. B. Saunders, 1976, p 539.

- Fuller DG, Hutton WL: Presurgical Evaluation of Eyes with Opaque Media. London, Grune \& Stratton, 1982.

5 Wendel RT, Mannis MJ, Keltner JL: Role of electrophysiologic testing in the preoperative evaluation of corneal transplant patients. Ann Ophthalmol 1984;16:788-793.

-6 Heckenlively JR, Arden GB: Principles and Practice of Clinical Electrophysiology of Vision, ed 2. Cambridge, The MIT Press, 2006.

7 Ray S, Khan BF, Dohlman CH, D'Amico DJ: Management of vitreoretinal complications in eyes with permanent keratoprosthesis. Arch Ophthalmol 2002;120:559-566.

-8 Zerbe BL, Belin MW, Ciolino JB; Boston Type 1 Keratoprosthesis Study Group: Results from the multicenter Boston Type 1 Keratoprosthesis Study. Ophthalmology 2006;113:1779.e1-e7.

-9 Ghaffariyeh A, Honarpisheh N, Karkhaneh A, Abudi R, Moroz ZI, Peyman A, Faramarzi A, Abasov F: Fyodorov-Zuev keratoprosthesis implantation: long-term results in patients with multiple failed corneal grafts. Graefes Arch Clin Exp Ophthalmol 2011;249:93-101.

10 Banitt M: Evaluation and management of glaucoma after keratoprosthesis. Curr Opin Ophthalmol 2011;22:133-136.

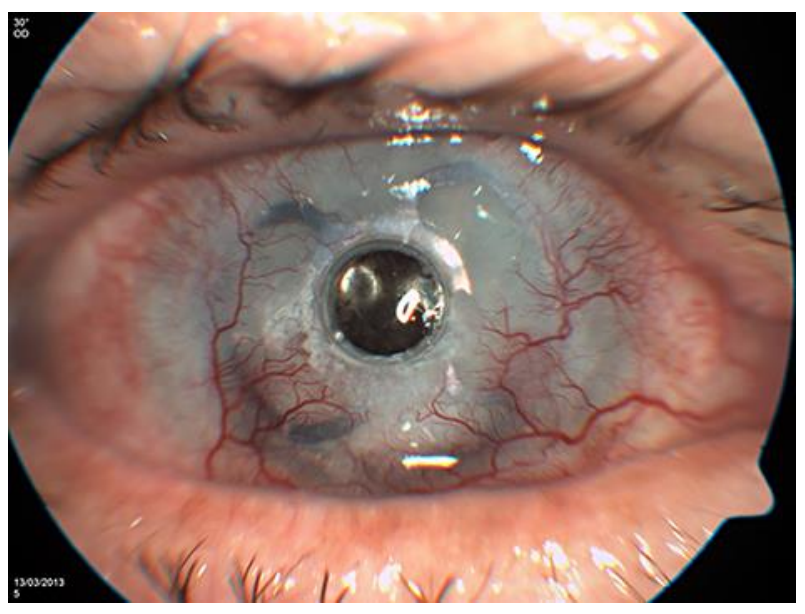

Fig. 1. RE. Note the Fyodorov-Zuev keratoprosthesis and corneal pannus. 
Schwartz et al.: Visually Evoked Potentials in a Patient with a Fyodorov-Zuev Keratoprosthesis

\section{2 - BOTH EYES - Flash VEP $1 \mathrm{cds} / \mathrm{m} 2$}
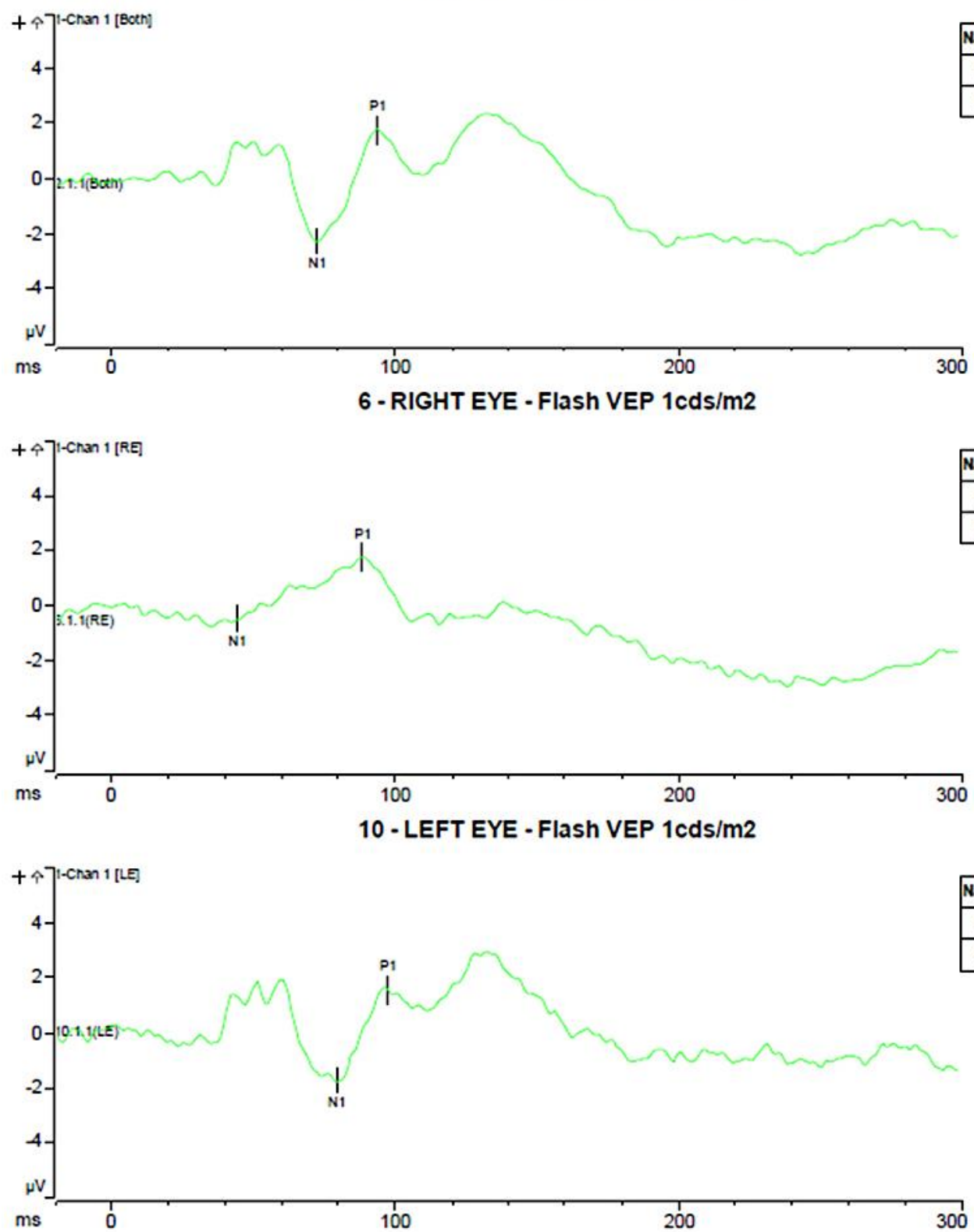

Fig. 2. Flash VEP prior to surgery. The top graph represents both eyes, the middle graph the RE, and the bottom graph a VEP of the LE. 
Case Reports in

Ophthalmology

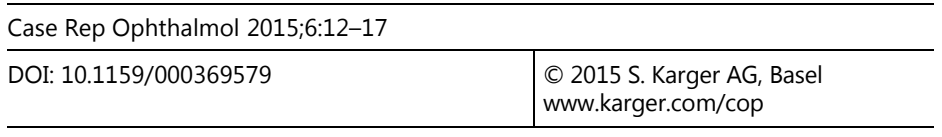

Schwartz et al.: Visually Evoked Potentials in a Patient with a Fyodorov-Zuev Keratoprosthesis

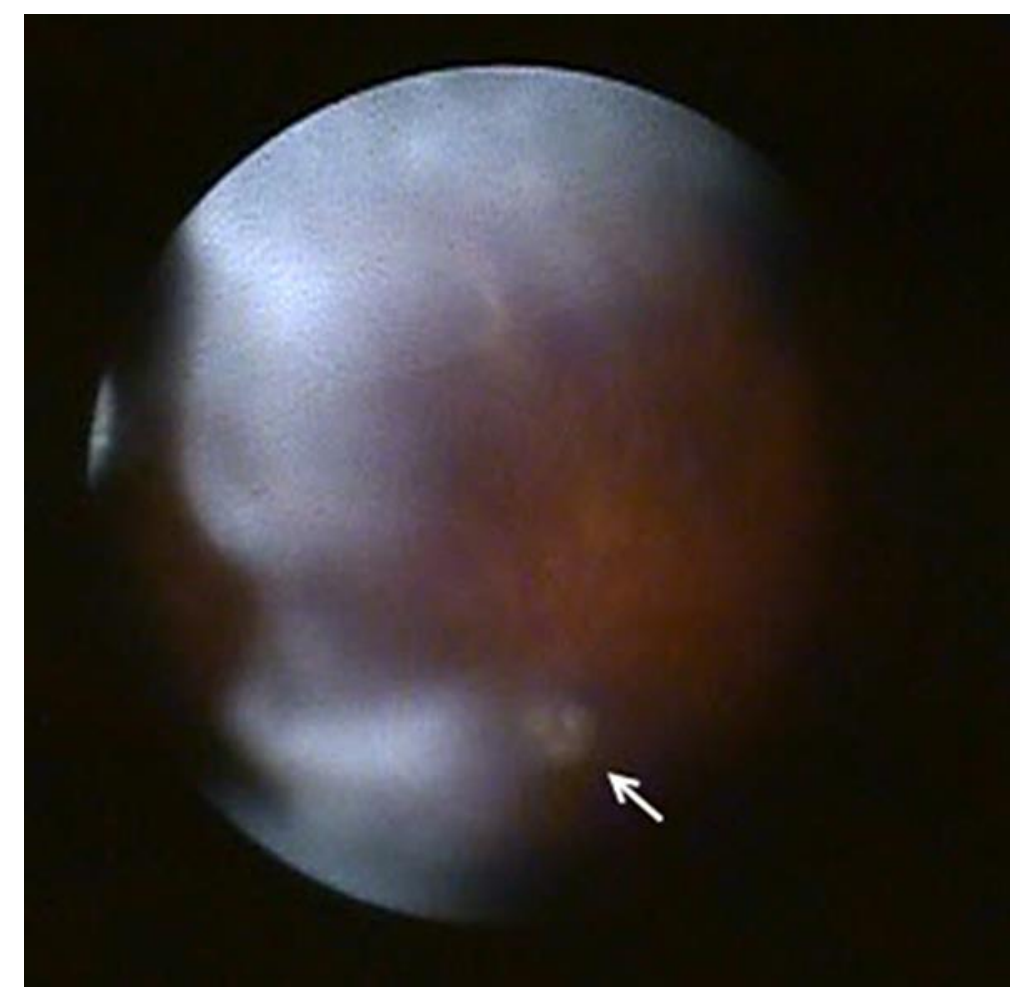

Fig. 3. Vitreal opacities hiding a normal looking retina, as seen during surgery. Notice the pale disc with an increased C/D ratio (arrow). 